\title{
$\mathrm{CuCl}_{2}$ 催化 “一锅法” 合成 2-苄基-3,4-二氢异喹啉-1-酮类衍生物
}

\author{
付超樊彦霞孙启辉易维银* 易封萍* \\ (上海应用技术大学香料香精技术与工程上海 201418)
}

\begin{abstract}
摘要 成功开发了一种简便、有效地由铜催化 “一锅法” 合成 2-芐基-3,4-二氢异喹啉-1-酮类化合物的方法. 该方法以 四氢异咺啉、苄基叠氮、含取代基的苯甲醛为原料，经过亲核加成并进一步氧化反应，一锅法合成目标化合物，且目标 化合物产率为 $50 \% \sim 70 \%$. 同时，进一步探究了催化剂的种类、添加剂的种类、反应温度、投料比等因素对反应的影响. 结果表明, 在 $\mathrm{CuCl}_{2}$ 为催化剂, $\mathrm{CH}_{3} \mathrm{COOH}$ 为添加剂, 甲苯为溶剂的条件下, 可高效地得到一系列 2-芐基-3,4-二氢异喹 啉-1-酮类化合物.
\end{abstract}

关键词 氯化铜; 一锅法; 2-芐基-3,4-二氢异喹啉-1-酮

\section{One-Pot Synthesis of 2-Benzyl-3,4-dihydro-2H-isoquinolin-1-ones Catalyzed by $\mathrm{CuCl}_{2}$}

\author{
Fu, Chao Fan, Yanxia Sun, Qihui Yi, Weiyin* Yi, Fengping* \\ (School of Perfume and Aroma Technology, Shanghai Institute of Technology, Shanghai 201418)
}

\begin{abstract}
An facile, effective, copper-catalyzed one-pot approach to 2-benzyl-3,4-dihydroquinolin-1-ones has been successfully developed. The compounds were achieved by the reaction of tetrahydroisoquinoline, benzyl azide and benzaldehyde in one-pot fashion via nucleophilic addition reaction followed by oxidation. The desired products were afforded in the high yield of $50 \% \sim 70 \%$. Moreover, The effects of different catalyst, additives, reaction temperature and proportion of substrates on the reaction were also investigated. The results showed that the optimizing conditions of reaction were $\mathrm{CuCl}_{2}$ as catalyst, $\mathrm{CH}_{3} \mathrm{COOH}$ as additive and toluene as solvent. A series of 2-benzyl-3, 4-dihydro- $2 \mathrm{H}$-isoquinolin-1-ones can be efficiently obtained under the optimizing conditions.
\end{abstract}

Keywords $\mathrm{CuCl}_{2}$; one-pot; 2-benzyl-3,4-dihydro-2 $H$-isoquinolin-1-ones

四氢异喹啉类化合物不仅是一种天然的含氮杂环 类化合物, 也是重要的生物活性药物 ${ }^{[1]}$. 四氢异喹啉及 其衍生物被广泛地应用于抗肿瘤 ${ }^{[2]}$ 、抗真菌 ${ }^{[3]}$ 、抗 HV 病毒 ${ }^{[4]} 、$ 抗凝血 ${ }^{[5]}$ 等药物中. 作为四氢异喹啉类化合物的 重要组成部分, 二氢异喹啉酮及其衍生物在许多天然生 物活性物质中作为核心结构存在 ${ }^{[6]}$, 同时也是各种天然 产物合成中的关键中间体 ${ }^{[7]}$. 因此, 探索二氢异喹啉酮 类化合物的有效合成方法具有重要的研究意义.

在四氢异喹啉类化合物中, 二氢异喹啉酮类化合物 的合成研究报道较多, 其合成方法主要分为以下几种类 型. $\mathrm{Li}$ 等 $^{[8]}$ 报道了通过固相合成方法得到相应的异喹啉 铜类化合物. 整个合成过程不仅需 $5 \sim 6$ 步进行, 且多步 反应产生大量的废弃物; Ban 等 ${ }^{[9]}$ 在 $\mathrm{NaBH}_{4}$ 条件下, 通 过 2-溴苯乙胺与苯甲醛反应合成了二氢异喹啉酮类化
合物, 该反应需要一氧化碳的参与才能发生, 而一氧化 碳是一种有毒气体, 对人体有害. 近年来, 有研究报道, 直接使用四氢异喹啉类化合物为底物, 选择性 $\alpha$ 位氧化 得到相应的二氢异喹啉酮. 但大多数反应需要使用昂贵 且有毒的过渡金属催化剂(如金 ${ }^{[10]}$ 、钓 ${ }^{[1]}$ 等)和 $\mathrm{I}_{2}{ }^{[12]}$ 、高

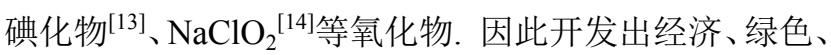
环保的二氢异喹啉合成方法具有重大意义.

根据文献调查发现，尚未有报道在四氢异喹啉及衍 生物的 1 位碳上，通过一锅的亲核加成及氧化引入羰基 直接合成二氢异喹啉酮类化合物. 因此我们在以往研究 基础上，进一步开发出了在铜催化剂和冰乙酸条件下， 使用四氢异喹啉、苯甲醛、苄基叠氮作底物, 直接一步 合成 2-芐基-3,4-二氢异喹啉-1-酮. 同时, 对其反应条 件、催化剂体系、最佳反应温度、最佳投料比进行了一

\footnotetext{
* Corresponding author. E-mail: yifengping@sit.edu.cn

Received May 10, 2018; revised July 24, 2018; published online September 5, 2018.
} 
系列系统地探索与优化. 该方法原料易得、操作简单、 产率较高，具有较大应用价值.

\section{1 结果与讨论}

为了得到优化的反应条件, 实验过程中以四氢异喹 啉(1)、苯甲醛(2a)、芐基叠氮(3)一锅法反应为模板反应, 考察了催化剂的种类、底物比以及反应温度对反应的影 响，具体结果如表 1 所示.

表 1 目标产物合成反应条件的优化 ${ }^{a}$

Table 1 Optimization of reaction conditions

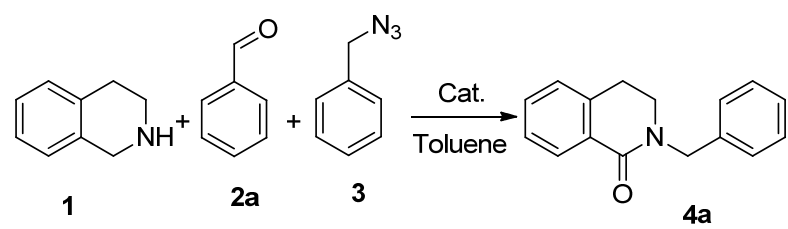

\begin{tabular}{clcc}
\hline Entry & Catalyst & Temp. $/{ }^{\circ} \mathrm{C}$ & Yield/\% \\
\hline 1 & $\mathrm{CuI}$ & 100 & 31 \\
2 & $\mathrm{CuBr}$ & 100 & 23 \\
3 & $\mathrm{CuCl}$ & 100 & 21 \\
4 & $\mathrm{CuBr}_{2}$ & 100 & 41 \\
5 & $\mathrm{CuCl}_{2}$ & 100 & 60 \\
6 & $\mathrm{FeCl}_{3} \cdot 6 \mathrm{H}_{2} \mathrm{O}$ & 100 & 23 \\
7 & $\mathrm{Fe}_{2}\left(\mathrm{SO}_{4}\right)_{3}$ & 100 & 19 \\
8 & $\mathrm{Cu}(\mathrm{OTf})_{2}$ & 100 & 0 \\
9 & $\mathrm{Ag}(\mathrm{OTf})$ & 100 & 42 \\
10 & $\mathrm{Bi}(\mathrm{OTf})_{3}$ & 100 & 14 \\
11 & $\mathrm{Ce}(\mathrm{OTf})_{3}$ & 100 & 9 \\
12 & ${\mathrm{Yb}(\mathrm{OTf})_{3}}$ & 100 & 0 \\
13 & $\mathrm{CuCl}_{2}$ & 100 & $41^{b}, 46^{c}, 52^{d}$ \\
14 & $\mathrm{CuCl}_{2}$ & 80 & 46 \\
15 & $\mathrm{CuCl}_{2}$ & 60 & 43 \\
16 & $\mathrm{CuCl}_{2}$ & 110 & 52 \\
17 & $\mathrm{CuCl}_{2}$ & 120 & 50 \\
\hline
\end{tabular}

${ }^{a}$ Reaction condition: 1 (1.4 mmo1), 2a $(1.4 \mathrm{mmol}), \mathbf{3}(1 \mathrm{mmol})$, cat. $(10$ mol\%), toluene $(5 \mathrm{~mL}), \mathrm{CH}_{3} \mathrm{COOH}(12 \mu \mathrm{L}), 100{ }^{\circ} \mathrm{C}, 3 \mathrm{~h}$. The molar ratio: ${ }^{b} \mathbf{1}: \mathbf{2 a}: \mathbf{3}=1: 1: 1 ;{ }^{c} \mathbf{1}: \mathbf{2 a}: \mathbf{3}=1.2: 1.2: 1 ;{ }^{d} \mathbf{1}: \mathbf{2 a}: \mathbf{3}=1.8: 1.8: 1$.

首先，考察了不同催化剂对反应的影响. 实验结果 表明不同金属催化剂对反应产率的影响不同(表 1, Entries 1 12). 在一价铜催化剂中, $\mathrm{CuBr}$ 和 $\mathrm{CuCl}$ 的催化效 果基本相同, 而 $\mathrm{CuI}$ 的催化效果要好于 $\mathrm{CuBr}$ 和 $\mathrm{CuCl}$ 的 催化效果(表 1, Entries 1 3); 在二价铜催化剂中, $\mathrm{CuCl}_{2}$ 的催化效果明显好于 $\mathrm{CuBr}_{2}$, 产率达到 $60 \%$ (表 1, Entry 5). 在铁的催化剂中, 测定了 $\mathrm{FeCl}_{3} \cdot 6 \mathrm{H}_{2} \mathrm{O}$ 与 $\mathrm{Fe}_{2}\left(\mathrm{SO}_{4}\right)_{3}$ 的催化效果, 通过对比发现两者的催化效果不太显著, 而且催化效果明显低于二价铜催化剂(表 1, Entries 6 7). 同时, 还对铜、银、铋、铈、镱的三氟甲磺酸盐的 催化效果进行测定, 发现三氟甲磺酸铜与三氟甲磺酸镱 基本上对该反应没有催化效果(表 1, Entry 8，12)，三氟 甲磺酸铈和三氟甲磺酸铋的催化效果也较弱, 产率分别
仅有 9\%(表 1, Entry 11)和 14\%(表 1, Entry 10)，但三氟甲 磺酸银对该反应的催化效果较好，达到了 $42 \%$ 的产率 (表 1, Entry 9). 接下来, 考察了不同底物比对反应产率 的影响，设定了 3 组不同投料比(表 1, Entry 13). 从中可 以看出, 当物质的量比四氢异喹啉: 苯甲醛: 芐基叠氮 $=1.4: 1.4: 1$ 时, 为最佳投料比.

确定了最佳投料比后，本研究设计了不同反应温度 对反应产率的影响. 将投料比固定为 $1.4: 1.4: 1$, 设置 5 个梯度温度, 分别为 $60 、 80 、 110$ 和 $120{ }^{\circ} \mathrm{C}$ (表 1 , Entries $14 \sim 17)$, 以及上组中的 $100{ }^{\circ} \mathrm{C}$ (表 1 , Entry 5). 可以看出 随着温度从 $60{ }^{\circ} \mathrm{C}$ 升到 $80{ }^{\circ} \mathrm{C}$, 再到 $100{ }^{\circ} \mathrm{C}$, 产率逐步 提高, 在 $100{ }^{\circ} \mathrm{C}$ 时催化效果达到最好. 但随着温度继续 升高, 催化效果又逐渐降低并且出现更多的副产物. 最 终确定在 $\mathrm{CuCl}_{2}$ 作催化剂下, 该反应的最佳反应温度为 $100{ }^{\circ} \mathrm{C}$.

通过模版反应的研究，确定了最优的反应条件：四 氢异喹啉 $(1,1.4 \mathrm{mmol}) 、$ 苯甲醛 $(2 \mathrm{a}, 1.4 \mathrm{mmol}) 、$ 芐基叠 氮 $(3,1 \mathrm{mmol}) 、 \mathrm{CuCl}_{2}(10 \mathrm{~mol} \%)$ 和 $\mathrm{CH}_{3} \mathrm{COOH}(12 \mu \mathrm{L})$, 以甲苯为溶剂, 并加入 $4 \AA$ 分子篮 $\left(50 \mathrm{mg} \cdot \mathrm{mL}^{-1}\right), 100{ }^{\circ} \mathrm{C}$ 下反应 $3 \mathrm{~h}$. 在此优化条件下，选择了一系列的苯甲醛 类化合物与四氢异喹啉和芐基叠氮进行 “一锅法” 反应, 实验结果见表 2 .

首先，考察了苯环上取代基的位置对反应产率的影 响(表 2, Entries 2 4), 实验结果发现邻、间、对位氯取 代的苯甲醛产率相差不大，表明取代基的位置对该反应 的影响较小. 同时发现当苯环上有不同卤素取代时，如 氯、溴，澳取代的苯甲醛产率较高，达到 $62 \%$ (表 2, Entry 5 ). 当苯环上含有多个吸电子取代基时，该反应产率得 到进一步提高，最高可达到 70\%(表 2, Entries 6 8). 含 供电子取代的苯甲醛，如对甲基或对乙基苯甲醛在标准 条件下反应，分别以 $52 \%$ 和 $56 \%$ 的分离产率得到相应的 目标化合物(表 2, Entries 9 10). 同时含有吸电子基团 和供电子基团苯甲醛进行反应时，收率为 $58 \%$ (表 2 , Entry 11). 这说明无论苯环上连有供电子基还是吸电子 基, 反应均能进行, 苯环上电子云密度的变化对反应收 率影响很小. 比较而言, 吸电子基团的效果好于供电子 基团. 为进一步拓展反应的底物的适应范围, 考察了其 他杂芳环醛类底物. 糠醛被用于反应时, 可获得目标产 物, 产率为 54\%(表 2, Entry 12). 综上可知, 此方法适用 于不同结构类型和不同取代的 2-苄基-3,4-二氢异喹啉1-酮类化合物的合成.

在实验结果的基础上，对该反应过程机理进行了简 单推测(如图 1 所示). 在冰乙酸的作用下, 原料 $\mathbf{1}$ 和原料 $\mathbf{2}$ 初步反应生成中间产物 $\mathbf{A}$ ，接着脱去一分子水生成 $\mathbf{B}$; 产物 $\mathbf{B}$ 发生电子转移得到 $\mathbf{C}$, 中间产物 $\mathbf{C}$ 经亲核加成生 
表 2 “一锅法” 合成的适用性研究

Table 2 Applicability study of “one-pot” synthesis

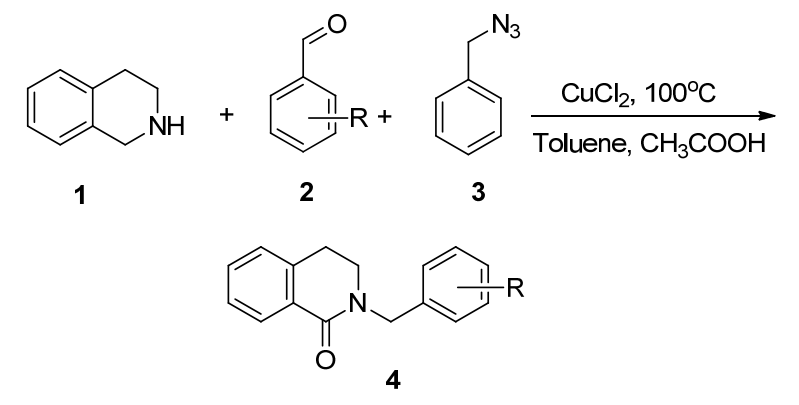

(2)

成 $\mathbf{D} ; \mathbf{D}$ 在铜盐的催化下，与苄基叠氮发生氧化反应，生 成最终产物 4, 同时并产生副产物茮胺, 其茮胺已通过 GC-MS 检测到.

\section{2 结论}

综上所述，通过四氢异喹啉、苯甲醛类和芐基叠氮 的一锅法反应，成功地开发了一种新的 $\mathrm{CuCl}_{2}$ 催化的四 氢异喹啉类衍生物的合成方法. 相较于其他合成方法, 该一锅法的特点与底物的来源方便性在二氢异喹啉酮 的合成上具有更广泛的适用性和优势. 此反应为合成四 氢异喹啉衍生物提供了一种高效、便捷的途径. 同时, 本文也为此类反应提出了合理的机制.

\section{3 实验部分}

\section{1 仪器与试剂}

${ }^{1} \mathrm{H}$ NMR 和 ${ }^{13} \mathrm{C}$ NMR 采用 AVANCE III 500M 核磁 共振仪测定, 以 $\mathrm{CDCl}_{3}$ 为溶剂, ${ }^{1} \mathrm{H} \mathrm{NMR}$ 以 $\mathrm{TMS}$ 或 $\mathrm{CHCl}_{3}$ 为标准. 高分辨质谱仪采用 solanX 70 FT-MS 测 定. 熔点测定仪采用 Yanaco micrometer 显微熔点测定 仪(温度未经校正)。原料芐基叠氮参考相应文献合成 ${ }^{[15]}$, 其他实验试剂均为市售分析纯试剂，未进一步纯化处 理.

\section{2 实验方法}

\subsection{1 苄基叠氮的制备}

将苠溴 $(10 \mathrm{mmol})$ 和 DMF $(20 \mathrm{~mL})$ 加入到 $50 \mathrm{~mL}$ 的双 颈圆底烧瓶中, 搅拌下加入叠氮化钠 $(11 \mathrm{mmol})$, 在氮气 条件下, 将该溶液在室温下搅拌 $24 \mathrm{~h}$. 反应结束后, 反 应液转入分液漏斗中, 加入 $50 \mathrm{~mL}$ 乙酸乙酯和 $50 \mathrm{~mL}$ 水, 振摇分液, 分出水相, 有机相用水洗涤 $(15 \mathrm{~mL} \times 3)$, 再 用饱和食盐水洗涤一次, 无水硫酸钠干燥. 滤除干燥剂 后, 通过旋转蒸发仪除去有机溶剂, 残余物通过柱层析 分离 $[V$ (石油醚)： $V$ (乙酸乙酯 $)=10 ： 1]$, 最终得到 $1.2 \mathrm{~g}$ 苄基叠氮, 为黄色液体, 产率为 $90 \%$.

\subsubsection{2-荬基-3,4-二氢异喹啉-1-酮类化合物的合成}

以产物 2-芐基-3,4-二氢异喹啉-1-酮(4a)的合成为 例. 将 $\mathrm{CuCl}_{2}(14 \mathrm{mg}, 0.1 \mathrm{mmol}) 、$ 冰乙酸 $(12 \mu \mathrm{L})$ 、活化后 的分子篮 $(4 \AA, 200 \mathrm{mg}$ )一次性加入圆底烧瓶, 加入溶剂 甲苯 $(5 \mathrm{~mL})$ 之后, 紧接着加入四氢异喹啉(186 $\mathrm{mg}, 1.4$ $\mathrm{mmol}$ )、苯甲醛(148 mg, $1.4 \mathrm{mmol}$ )、苄基叠氮(133 mg, 1 $\mathrm{mmol})$. 然后把反应温度控制到 $100{ }^{\circ} \mathrm{C}$ 反应 $3 \mathrm{~h}$. 反应进 程使用薄层色谱 $(\mathrm{TLC})$ 监测 $[V$ (石油醚) $: V$ (乙酸乙酯 $)=$ $10 ： 1]$. 反应结束后, 用镊子取出其中的 $4 \AA$ 分子篮并 用二氯甲烷淋洗 3 4 次, 将所得溶液通过旋转蒸发仪 除去有机溶剂，残余物使用快速柱层析方法纯化分离 


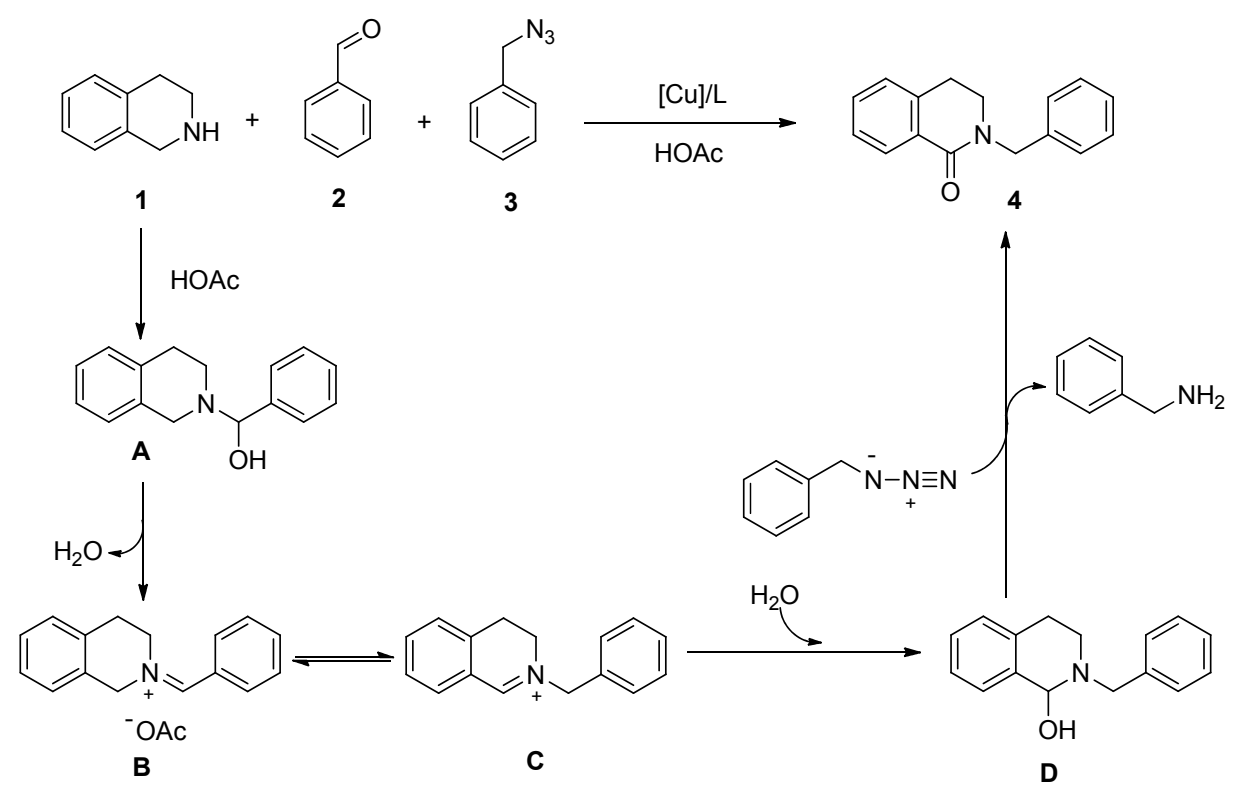

图 1 “一锅法” 合成的机理探讨

Figure 1 Reaction mechanism of "one-pot" synthesis

$[V($ 石油醚 $): V($ 乙酸乙酯 $)=10: 1]$, 最后得到 $0.14 \mathrm{~g}$ 浅 黄色液体 2-茮基-3,4-二氢异喹啉-1-酮(4a), 产率为 $60 \%$. ${ }^{1} \mathrm{H}$ NMR $\left(500 \mathrm{MHz}, \mathrm{CDCl}_{3}\right) \delta: 8.18(\mathrm{~d}, J=7.6 \mathrm{~Hz}, 1 \mathrm{H})$, 7.45 (t, $J=6.9 \mathrm{~Hz}, 1 \mathrm{H}), 7.39$ (d, $J=7.5 \mathrm{~Hz}, 1 \mathrm{H}), 7.36$ (d, $J=4.5 \mathrm{~Hz}, 2 \mathrm{H}), 7.31$ (s, 1H), 7.19 (d, $J=7.4 \mathrm{~Hz}, 1 \mathrm{H}), 4.83$ (s, 2H), $3.51(\mathrm{~s}, 1 \mathrm{H}), 2.96(\mathrm{~s}, 1 \mathrm{H}) ;{ }^{13} \mathrm{C} \mathrm{NMR}(125 \mathrm{MHz}$, $\left.\mathrm{CDCl}_{3}\right) \delta: 164.6,138.1,137.5,131.7,129.4,128.6,128.5$, 128.1, 127.4, 127.1, 126.9, 50.5, 45.4, 28.1; HRMS calcd for $\mathrm{C}_{16} \mathrm{H}_{16} \mathrm{NO}[\mathrm{M}+\mathrm{H}]^{+}$238.1984, found 238.1986.

2-(2-氯-苄基)-3,4-二氢异喹啉-1-酮(4b): 浅黄色液 体, 产率 53\%. ${ }^{1} \mathrm{H}$ NMR $\left(500 \mathrm{MHz}, \mathrm{CDCl}_{3}\right) \delta: 8.14$ (d, $J=$ $7.5 \mathrm{~Hz}, 1 \mathrm{H}), 7.44$ (t, $J=7.5 \mathrm{~Hz}, 1 \mathrm{H}), 7.40 \sim 7.34(\mathrm{~m}, 3 \mathrm{H})$, $7.25 \sim 7.20(\mathrm{~m}, 2 \mathrm{H}), 7.18(\mathrm{~d}, J=7.0 \mathrm{~Hz}, 1 \mathrm{H}), 4.92(\mathrm{~s}, 2 \mathrm{H})$, $3.55(\mathrm{t}, J=6.5 \mathrm{~Hz}, 2 \mathrm{H}), 2.99$ (t, $J=6.5 \mathrm{~Hz}, 2 \mathrm{H}) ;{ }^{13} \mathrm{C} \mathrm{NMR}$ $\left(125 \mathrm{MHz}, \mathrm{CDCl}_{3}\right) \delta: 164.8,138.1,134.8,133.6,131.9$, $129.6,129.5,129.2,128.7,128.5,127.2,127.1,126.9$, 48.1, 45.9, 28.1; HRMS calcd for $\mathrm{C}_{16} \mathrm{H}_{15} \mathrm{CINO}[\mathrm{M}+\mathrm{H}]^{+}$ 272.0836 , found 272.0839 .

2-(3-氯-苠基)-3,4-二氢异喹啉-1-酮(4c)：浅黄色液 体, 产率为 $50 \%$. ${ }^{1} \mathrm{H}$ NMR $\left(500 \mathrm{MHz}, \mathrm{CDCl}_{3}\right) \delta: 8.14$ (d, $J=8.0 \mathrm{~Hz}, 1 \mathrm{H}), 7.44$ (t, $J=7.0 \mathrm{~Hz}, 1 \mathrm{H}), 7.37$ (t, $J=7.5$ $\mathrm{Hz}, 1 \mathrm{H}), 7.32$ (s, 1H), 7.25 (t, $J=7.0 \mathrm{~Hz}, 4 \mathrm{H}), 7.18$ (d, $J=$ $7.5 \mathrm{~Hz}, 1 \mathrm{H}), 4.77$ (s, 2H), 3.50 (t, $J=6.5 \mathrm{~Hz}, 2 \mathrm{H}), 2.97$ (t, $J=7.0 \mathrm{~Hz}, 2 \mathrm{H}) ;{ }^{13} \mathrm{C} \mathrm{NMR}\left(125 \mathrm{MHz}, \mathrm{CDCl}_{3}\right) \delta: 164.7$, $139.6,138.0,134.6,131.9$, 129.9, 129.2, 128.5, 128.1, 127.7, 127.2, 127.0, 126.2, 50.1, 45.6, 28.1; HRMS calcd for $\mathrm{C}_{16} \mathrm{H}_{15} \mathrm{ClNO}[\mathrm{M}+\mathrm{H}]^{+}$272.0836, found 272.0841. 2-(4-氯一茮基)-3,4-二氢异喹啉-1-酮(4d): 浅黄色液 体, 产率为 $58 \% .{ }^{1} \mathrm{H} \mathrm{NMR}\left(500 \mathrm{MHz}, \mathrm{CDCl}_{3}\right) \delta: 8.13(\mathrm{~d}$, $J=7.5 \mathrm{~Hz}, 1 \mathrm{H}), 7.46 \sim 7.40(\mathrm{~m}, 1 \mathrm{H}), 7.36(\mathrm{t}, J=7.5 \mathrm{~Hz}$, $1 \mathrm{H}), 7.32 \sim 7.25(\mathrm{~m}, 3 \mathrm{H}), 7.17(\mathrm{~d}, J=7.5 \mathrm{~Hz}, 1 \mathrm{H}), 4.75(\mathrm{~s}$, $1 \mathrm{H}), 3.48(\mathrm{t}, J=6.5 \mathrm{~Hz}, 1 \mathrm{H}), 2.94(\mathrm{t}, J=6.5 \mathrm{~Hz}, 1 \mathrm{H}) ;{ }^{13} \mathrm{C}$ NMR $\left(125 \mathrm{MHz}, \mathrm{CDCl}_{3}\right) \delta: 164.7,138.0,136.0,133.3$, $131.9,129.5,129.2,128.8,128.5,127.2,127.0,49.9,45.5$, 28.1; HRMS calcd for $\mathrm{C}_{16} \mathrm{H}_{15} \mathrm{CINO}[\mathrm{M}+\mathrm{H}]^{+}$272.0836, found 272.0840 .

2-(2-溴-苄基)-3,4-二氢异喹啉-1-酮(4e)：黄色液体， 产率为 $62 \% .{ }^{1} \mathrm{H}$ NMR $\left(500 \mathrm{MHz}, \mathrm{CDCl}_{3}\right) \delta: 8.13(\mathrm{~d}, J=$ $7.6 \mathrm{~Hz}, 1 \mathrm{H}), 7.44(\mathrm{dd}, J=13.0,8.0 \mathrm{~Hz}, 3 \mathrm{H}), 7.36$ (t, $J=7.5$ $\mathrm{Hz}, 1 \mathrm{H}), 7.22$ (d, $J=8.0 \mathrm{~Hz}, 2 \mathrm{H}), 7.17$ (d, $J=7.5 \mathrm{~Hz}, 1 \mathrm{H})$, $4.74(\mathrm{~s}, 2 \mathrm{H}), 3.48(\mathrm{t}, J=6.5 \mathrm{~Hz}, 2 \mathrm{H}), 2.94(\mathrm{t}, J=6.5 \mathrm{~Hz}$, $2 \mathrm{H}) ;{ }^{13} \mathrm{C} \mathrm{NMR}\left(125 \mathrm{MHz}, \mathrm{CDCl}_{3}\right) \delta: 164.2,164.0,138.2$, $136.4,132.9,131.9,129.3,128.9,128.5,127.8,127.2$, 127.0, 123.7, 50.6, 45.9, 28.2; HRMS calcd for $\mathrm{C}_{16} \mathrm{H}_{15} \mathrm{BrNO}[\mathrm{M}+\mathrm{H}]^{+}$316.0331, found 316.0339.

2-(2,4-二氯-茮基)-3,4-二氢异喹啉-1-酮(4f)：黄色 固体，产率为 70\%. m.p. 95 98 ${ }^{\circ} \mathrm{C} ;{ }^{1} \mathrm{H}$ NMR $(500 \mathrm{MHz}$, $\left.\mathrm{CDCl}_{3}\right) \delta: 8.12(\mathrm{~d}, J=8.0 \mathrm{~Hz}, 1 \mathrm{H}), 7.44(\mathrm{t}, J=7.5 \mathrm{~Hz}, 1 \mathrm{H})$, $7.40(\mathrm{~d}, J=1.5 \mathrm{~Hz}, 1 \mathrm{H}), 7.38 \sim 7.34(\mathrm{~m}, 2 \mathrm{H}), 7.23 \sim 7.17$ $(\mathrm{m}, 2 \mathrm{H}), 4.87(\mathrm{~s}, 2 \mathrm{H}), 3.56(\mathrm{t}, J=6.5 \mathrm{~Hz}, 2 \mathrm{H}), 2.99(\mathrm{t}, J=$ $6.5 \mathrm{~Hz}, 2 \mathrm{H}) ;{ }^{13} \mathrm{C}$ NMR $\left(125 \mathrm{MHz}, \mathrm{CDCl}_{3}\right) \delta: 164.9,138.1$, $134.2,133.8,133.6,132.0,130.5,129.37,129.1,128.5$, 127.5, 127.2, 127.0, 47.8, 46.2, 28.2; HRMS calcd for $\mathrm{C}_{16} \mathrm{H}_{14} \mathrm{Cl}_{2} \mathrm{NO}[\mathrm{M}+\mathrm{H}]^{+}$306.0446, found 306.0451. 
2-(2-溴-4-氯-芐基)-3,4-二氢异喹啉-1-酮 (4g)：黄色 固体, 产率为 $60 \%$. m.p. $98 \sim 100{ }^{\circ} \mathrm{C} ;{ }^{1} \mathrm{H}$ NMR $(500$ $\left.\mathrm{MHz}, \mathrm{CDCl}_{3}\right) \delta: 8.13(\mathrm{~d}, J=7.5 \mathrm{~Hz}, 1 \mathrm{H}), 7.59$ (d, $J=2.0$ $\mathrm{Hz}, 1 \mathrm{H}), 7.45$ (t, $J=7.5 \mathrm{~Hz}, 1 \mathrm{H}), 7.37$ (t, $J=7.5 \mathrm{~Hz}, 1 \mathrm{H}$ ), $7.32(\mathrm{~d}, J=8.0 \mathrm{~Hz}, 1 \mathrm{H}), 7.28 \sim 7.24(\mathrm{~m}, 1 \mathrm{H}), 7.19(\mathrm{~d}, J=$ $7.5 \mathrm{~Hz}, 1 \mathrm{H}), 4.86(\mathrm{~s}, 2 \mathrm{H}), 3.55$ (t, $J=6.5 \mathrm{~Hz}, 2 \mathrm{H}), 3.00$ (t, $J=6.5 \mathrm{~Hz}, 2 \mathrm{H}) ;{ }^{13} \mathrm{C}$ NMR $\left(125 \mathrm{MHz}, \mathrm{CDCl}_{3}\right) \delta: 164.9$, $138.1,135.2,133.9,132.5,132.0,130.3,129.1,128.5$, 128.0, 127.2, 127.0, 123.8, 50.1, 46.1, 28.2; HRMS calcd for $\mathrm{C}_{16} \mathrm{H}_{14} \mathrm{BrClNO}[\mathrm{M}+\mathrm{H}]^{+}$349.9941, found 349.9959.

2-(2-澳-5-氟-芐基)-3,4-二氢异喹啉-1-酮(4h)：淡黄 色固体, 产率为 $65 \%$. m.p. $108 \sim 110{ }^{\circ} \mathrm{C} ;{ }^{1} \mathrm{H}$ NMR (500 $\left.\mathrm{MHz}, \mathrm{CDCl}_{3}\right) \delta: 8.14(\mathrm{~d}, J=7.5 \mathrm{~Hz}, 1 \mathrm{H}), 7.52(\mathrm{dd}, J=9.0$, $5.0 \mathrm{~Hz}, 1 \mathrm{H}), 7.48 \sim 7.43(\mathrm{~m}, 1 \mathrm{H}), 7.38(\mathrm{t}, J=7.5 \mathrm{~Hz}, 1 \mathrm{H})$, 7.20 (d, $J=7.5 \mathrm{~Hz}, 1 \mathrm{H}), 7.10 \sim 7.05$ (m, 1H), 6.87 (td, $J=$ $8.5,3.0 \mathrm{~Hz}, 1 \mathrm{H}), 4.86$ (s, 2H), 3.58 (t, $J=6.5 \mathrm{~Hz}, 2 \mathrm{H}), 3.03$ (t, $J=6.5 \mathrm{~Hz}, 2 \mathrm{H}) ;{ }^{13} \mathrm{C} \mathrm{NMR}\left(125 \mathrm{MHz}, \mathrm{CDCl}_{3}\right) \delta: 164.9$, $161.4,138.7,138.1,134.1,132.0,129.0,128.6,127.2$, 127.1, 117.4, 116.2, 116.0, 50.8, 46.3, 28.2; HRMS calcd for $\mathrm{C}_{16} \mathrm{H}_{14} \mathrm{BrFNO}[\mathrm{M}+\mathrm{H}]^{+}$334.0237, found 334.0239.

2-(4-甲基-芐基)-3,4-二氢异喹啉-1-酮(4i): 淡黄色 液体, 产率为 $52 \% .{ }^{1} \mathrm{H}$ NMR $\left(500 \mathrm{MHz}, \mathrm{CDCl}_{3}\right) \delta: 8.14$ (d, $J=7.5 \mathrm{~Hz}, 1 \mathrm{H}), 7.41$ (t, $J=7.0 \mathrm{~Hz}, 1 \mathrm{H}), 7.35$ (t, $J=7.5$ $\mathrm{Hz}, 1 \mathrm{H}), 7.22$ (d, $J=8.0 \mathrm{~Hz}, 2 \mathrm{H}), 7.14$ (t, $J=7.5 \mathrm{~Hz}, 3 \mathrm{H})$, 4.75 (s, 2H), 3.47 (t, $J=6.5 \mathrm{~Hz}, 2 \mathrm{H}), 2.93$ (t, $J=6.5 \mathrm{~Hz}$, 2H), $2.33(\mathrm{~s}, 3 \mathrm{H}) ;{ }^{13} \mathrm{C}$ NMR (125 MHz, $\left.\mathrm{CDCl}_{3}\right) \delta: 164.6$, 138.1, 137.1, 134.4, 131.7, 129.5, 129.3, 128.5, 128.1, 127.1, 126.9, 50.2, 45.2, 28.1, 21.1; HRMS calcd for $\mathrm{C}_{17} \mathrm{H}_{18} \mathrm{NO}[\mathrm{M}+\mathrm{H}]^{+}$252.1382, found 252.1386.

2-(4-乙基-芐基)-3,4-二氢异喹啉-1-酮(4j)：淡黄色 液体, 产率为 $56 \% .{ }^{1} \mathrm{H}$ NMR $\left(500 \mathrm{MHz}, \mathrm{CDCl}_{3}\right) \delta: 8.17$ (d, $J=8.0 \mathrm{~Hz}, 1 \mathrm{H}), 7.44$ (t, $J=7.5 \mathrm{~Hz}, 1 \mathrm{H}), 7.38$ (t, $J=7.5$ $\mathrm{Hz}, 1 \mathrm{H}), 7.28$ (d, $J=8.0 \mathrm{~Hz}, 2 \mathrm{H}), 7.19(\mathrm{~d}, J=8.0 \mathrm{~Hz}, 3 \mathrm{H})$, 4.79 (s, 2H), 3.51 (t, $J=6.5 \mathrm{~Hz}, 2 \mathrm{H}), 2.95$ (t, $J=6.5 \mathrm{~Hz}$, $2 \mathrm{H}), 2.66(\mathrm{q}, J=7.5 \mathrm{~Hz}, 2 \mathrm{H}), 1.25(\mathrm{t}, J=7.5 \mathrm{~Hz}, 3 \mathrm{H}) ;{ }^{13} \mathrm{C}$ NMR $\left(125 \mathrm{MHz}, \mathrm{CDCl}_{3}\right) \delta: 164.6,143.5,138.1,134.6$, $131.7,129.5,128.5,128.1,127.1,126.9,50.2,45.3,28.5$, 28.1, 15.58; HRMS calcd for $\mathrm{C}_{18} \mathrm{H}_{20} \mathrm{NO}[\mathrm{M}+\mathrm{H}]^{+}$ 266.1539, found 266.1541 .

2-(2-澳-4-甲基-芐基)-3,4-二氢异喹啉-1-酮(4k)：淡 黄色液体, 产率为 $58 \% .{ }^{1} \mathrm{H}$ NMR $\left(500 \mathrm{MHz}, \mathrm{CDCl}_{3}\right) \delta$ : 8.17 (d, $J=7.5 \mathrm{~Hz}, 1 \mathrm{H}), 7.43$ (t, $J=7.5 \mathrm{~Hz}, 1 \mathrm{H}), 7.40$ (s, 1H), 7.36 (t, $J=7.5 \mathrm{~Hz}, 1 \mathrm{H}), 7.25$ (s, 1H), 7.18 (d, $J=7.4$ $\mathrm{Hz}, 1 \mathrm{H}), 7.08$ (d, $J=8.0 \mathrm{~Hz}, 1 \mathrm{H}), 4.88(\mathrm{~s}, 2 \mathrm{H}), 3.53$ (t, $J=$ $6.5 \mathrm{~Hz}, 2 \mathrm{H}), 2.97$ (t, $J=6.5 \mathrm{~Hz}, 2 \mathrm{H}), 2.31(\mathrm{~s}, 3 \mathrm{H}) ;{ }^{13} \mathrm{C}$
NMR (125 MHz, $\left.\mathrm{CDCl}_{3}\right) \delta: 164.7,139.1,138.2,133.3$, $131.8,129.4,129.3,128.6,128.5,127.1,127.0,123.5$, 45.7, 28.2, 20.7; HRMS calcd for $\mathrm{C}_{17} \mathrm{H}_{17} \mathrm{BrNO}[\mathrm{M}+\mathrm{H}]^{+}$ 330.0488 , found 330.0494 .

2-呋喃-2-亚甲基-3,4-二氢异喹啉-1-酮(4I)：棕色液 体, 产率为 $54 \% .{ }^{1} \mathrm{H}$ NMR $\left(500 \mathrm{MHz}, \mathrm{CDCl}_{3}\right) \delta: 8.13(\mathrm{~d}$, $J=7.5 \mathrm{~Hz}, 1 \mathrm{H}), 7.43(\mathrm{t}, J=7.5 \mathrm{~Hz}, 1 \mathrm{H}), 7.40 \sim 7.33(\mathrm{~m}$, $2 \mathrm{H}), 7.18(\mathrm{~d}, J=7.5 \mathrm{~Hz}, 1 \mathrm{H}), 6.37 \sim 6.32(\mathrm{~m}, 2 \mathrm{H}), 4.79(\mathrm{~s}$, $2 \mathrm{H}), 3.61(\mathrm{t}, J=6.5 \mathrm{~Hz}, 2 \mathrm{H}), 3.00(\mathrm{t}, J=6.5 \mathrm{~Hz}, 2 \mathrm{H}) ;{ }^{13} \mathrm{C}$ NMR $\left(125 \mathrm{MHz}, \mathrm{CDCl}_{3}\right) \delta: 164.4,151.0,142.3,138.1$, 131.8, 129.3, 128.5, 127.0, 126.9, 110.4, 108.4, 45.7, 43.3, 28.1; HRMS calcd for $\mathrm{C}_{14} \mathrm{H}_{14} \mathrm{NO}_{2}[\mathrm{M}+\mathrm{H}]^{+}$228.1019, found 228.1021 .

辅助材料(Supporting Information) 目标化合物 $4 \mathrm{a} \sim$ $4 \mathrm{I}$ 的 ${ }^{1} \mathrm{H}$ NMR, ${ }^{13} \mathrm{C}$ NMR 谱图. 这些材料可以免费从本 刊网站(http://sioc-journa1.cn/)上下载.

\section{References}

[1] (a) Siengalewicz, P.; Rinner, U.; Mulzer, J. Chem. Soc. Rev. 2008 , 2676.

(b) Campos, K. R.Chem. Soc. Rev. 2007, 36, 1069.

(c) Lebold, T. P.; Wood, J. L.; Deitch, J.; Lodewyk, M. W.; Tantillo, D. J.; Sarpong, R. Nat. Chem. 2013, 5, 126.

(d) Reddy, R. J.; Kawai, N.; Uenishi, J. J. Org. Chem. 2012, 77, 11101.

[2] Scott, J. D.; Williams, R. M. Chem. Rev. 2002, $102,1669$.

[3] Chandrasekhar, S.; Mohanty, P. K. Org. Lett. 1999, 1, 877.

[4] Kashiwada, Y.; Aoshima, A.; Ikeshiro, Y. Bioorg. Med. Chem. 2005, $13,443$.

[5] Zheng, M.; Zhang, X.; Zhao, M. Bioorg. Med. Chem. 2008, 16, 9574.

[6] (a) Fisher, M. J.; Gunn, B. P.; Harms, C. S.; Kline, A. D.; Mullaney, J. T. Bioorg. Med. Chem. Lett. 1997, 7, 2537.

(b) Jin, R.; Patureau, F. W. ChemCatChem 2015, 7, 223.

[7] (a) Gonzalez, D.; Martinot, T.; Hudlicky, T. Tetrahedron 1998, 40, 3077.

(b) McNulty, J.; Mao, J.; Gibe, R.; Mo, R.; Wolf, S.; Pettit, G. R.; Herald, D. L.; Boyd, M. R. Bioorg. Med. Chem. Lett. 2011, 11, 169. (c) Banwell, M. G.; Bissett, B. D.; Busato, S.; Cowden, C. J.; Hockess, D. C. R.; Holman, J. W.; Read, R. W.; Wu, A. W. J. Chem. Soc., Chem. Commun. 1995. 2551.

[8] Chern, M. S.; Li, W. R. Tetrahedron Lett. 2004, 45, 8323.

[9] Mori, M.; Chiba, K.; Ban, Y. J. Org. Chem. 1978, 43, 1684.

[10] (a) So, M. H.; Liu, Y. L.; Ho, C. M.; Che, C. M. Chem. Asian J. 2009, 4, 1551.

(b) Jin, X. J.; Kataoka, K.; Yatabe, T.; Yamaguchi, K.; Mizuno, N. Angew. Chem., Int. Ed. 2016, 55, 7212.

[11] Khusnutdinova, J. R.; Ben-David, Y.; Milstein, D. J. Am. Chem. Soc. 2014, 136, 2998.

[12] Griffiths, R.; Burly, G. A.; Talbot, E. P. A. Org. Lett. 2017,19, 870.

[13] Sueda, T.; Kajishima, D.; Goto, S. J. Org. Chem. 2003, 58, 3307.

[14] Song, A. R.; Yu, J.; Zhang, C. Synthesis 2012, 44, 2903.

[15] Alonso, F.; Moglie, Y.; Radivoy, G.; Yus, M. Eur. J. Org. Chem. 2010, 1875.

(Zhao, X.) 